\title{
Study protocol: systematic review and meta- analysis on the effectiveness of pharmacist intervention on health related quality of life and clinical outcomes among patients with heart failure
}

Mulubirhan Tirfe ( $\nabla$ mulubirhantirfe@gmail.com )

Aksum University https://orcid.org/0000-0002-7778-7898

Alemseged Beyene

Addis Ababa University College of Health Sciences

Haileselassie Berhane

Aksum University

Ephrem Engidawork

Addis Ababa University College of Health Sciences

Tewolde Teklu

Aksum University

\section{Protocol}

Keywords: Heart failure, Pharmacist intervention, Quality of life, Meta-analysis

Posted Date: May 22nd, 2020

DOI: https://doi.org/10.21203/rs.3.rs-28902/v1

License: (c) (i) This work is licensed under a Creative Commons Attribution 4.0 International License.

Read Full License 


\section{Abstract}

Background: Heart failure (HF) is associated with severe complications, hospitalization, and poor quality of life. Patients with heart failure had poor physical and emotional symptoms, functional status and worse health outcomes.

Objective: The aim of this systematic review and meta-analysis will be to investigate whether pharmacist intervention is effective in improving health related quality of life (HRQoL) and clinical outcomes among patients with heart failure.

Method: Systematic review and meta-analysis will be conducted. Published journals in English and indexed in Medline (PubMed), Embase, Cochrane Central Register of Controlled Trials (CENTRAL), Scopus, and Google scholar will be searched from 1990 to December 2019. Data will be extracted by one author and will be approved by other two authors independently. Data will be analyzed in accordance with the Cochrane handbook. Standardized mean differences will be used as an estimate of the effect size. Quality of included studies will be assessed using the modified Downs and Black checklist. Analysis for the dichotomous outcome studies will be converted into standardized mean difference and present with $95 \%$ confidence intervals. The review is approved in the International Prospective Register of Systematic Reviews (PROSPERO) with registration ID CRD42020158236.

Discussion: Currently there are important gaps on the effectiveness of pharmacist intervention in improving health-related quality of life and clinical outcomes. We believe this review will provide comprehensive evidence on the effectiveness of pharmacist intervention among patients with HF.

\section{Background}

Heart failure (HF) is a cardiac structural and/or functional abnormality leading to failure of the heart to deliver oxygen at a rate commensurate with the requirements of the metabolizing tissues [1]. Diagnosis of HF is confirmed based on clinical history, physical examination (Framingham criteria), chest X-ray and echocardiography findings and laboratory exams specifically plasma B-type Natriuretic Peptide and Nterminal B-type Natriuretic Peptide measurement [2, 3]. Clinical outcomes among patients with HF were mortality (all-cause and HF specific death), hospitalization, readmission and morbidity endpoints used in different trials and taken as definition by the European Society of Cardiology HF association consensus document [4].

Globally, about 26 million people were living with HF with poor patient outlook and worse survival [5]. HF put significant stress on patients, caregivers and the health care system. The five year mortality rate of systolic HF patients ranged from $25 \%$ to $75 \%$ due to sudden death from ventricular arrhythmia [6]. In a longitudinal study on community-living older persons from Cardiovascular Health Study, the rate of hospitalization was 7.9/10 person-years [7]. 
Multidisciplinary health professionals including nurses, pharmacists and dietitian's intervention on patient education, medication teaching and nutrition guidance, respectively, proved to have positive outcomes and lowered mortality [8]. Lower educational status was associated with an increased hospitalization of HF patients [9]. Low health literacy, which can be defined as Brief Health Literacy Screen $\leq 9$, was associated with higher risk of mortality and increased risk of hospital readmission [10]. Heart failure patients who have higher literacy have better understanding of their disease state, self efficacy and self care, thus patients with low literacy level were associated with worse HF related quality of life (QoL) [11].

Comparison of QoL among the healthy old age group versus ill old age HF patients showed a lesser QoL in the later ones. Using physical symptom as strongest measure, older adults with HF had poorer physical and emotional symptoms, poorer functional status and more worse health perception [12]. Using the German version generic QoL measure (SF-36) containing eight dimensions, scores of five of the eight QoL domains were reduced to around one-third in NYHA class III patients [13]. In the study conducted in Serbia, the poor QoL were due to lower income, longer history of chronic HF, longer hospital stay, multiple medications, higher NYHA class, depression and cognitive impairment [14].

Communicating hospitalized HF patients effectively at the time of discharge regarding their future clinical status improved QoL of patients [15]. In this case, the involvement of pharmacists demonstrated better medication adherence compared to other health professionals and had greater impact on the ability to inform, solve problem and support patients directly [16]. The pooled data of the systematic review and meta-analysis study on the pharmacist-led medication reconciliation programs, showed a significant reduction in adverse drug event-related hospital and emergency department visits, reduction in hospital readmissions and improved medication adherence $[17,18]$. Overall, pharmacists significantly improved health related quality of life (HRQoL) through pharmaceutical care interventions in terms of general health, social and physical functioning [19]

\section{Importance of this review}

Pharmacists can review medication charts and make interventions such as medication reconciliation for discharged patients and correct medication discrepancies, dealing with barriers to medication documentation and interdisciplinary communication [20]. Pharmacist-led medication review and reconciliation were effective in improving medication adherence and patient outcomes, as well as in reducing hospitalization improving post-hospital medication safety and health care utilization $[17,18]$. Pharmacists play significant roles in handling HF patients by minimizing disease symptoms, improving medication compliance and enhancing chronic disease management [21].

Clinical pharmacists can identify and resolve pharmaceutical care issues and provide optimal care when working in collaboration with other health care professionals [22]. Clinical pharmacists lower prescription errors and medication discrepancies through the discharge service to HF patients. The clinical pharmacist's activities includes: review of medications, communication with cardiologists, general 
practitioners and community pharmacists, providing patient information and preparing written overview of discharge medication [23].

Pharmacists decreased readmission of HF patients and improved care through continuum of care such as discharge counseling services and resolving medication reconciliation discrepancies. Patients discharged with the diagnosis of HF who received continuum of care had lower 30-day all-cause readmission rate [24].

Providing pharmaceutical care service to patients with HF had significant clinical and humanistic benefits. Pharmacist-led pharmaceutical care programs were shown to improve exercise tolerance (2-min walk test), forced vital capacity, medication adherence and HRQoL measured by the Minnesota living with heart failure questionnaire [25].

The systematic review and meta-analysis study demonstrated multidisciplinary interventions for HF reduced both hospital admission and all-cause mortality [26]. Pharmaceutical care interventions significantly improved HRQoL measures. However, the pooled data on HF- specific measures indicated no significant impact of pharmaceutical care utilization [19]. Therefore the aim of this systematic review and meta-analysis is to investigate whether pharmacist intervention is effective on quality of life and clinical outcomes among patients with HF.

\section{Objective}

The aim of this study protocol is to provide a clear way to review systematically from various indexing databases and synthesize the data that whether pharmacist intervention is effective on HRQoL and clinical outcomes compared to usual/standard care. This review will address systematic literature search strategy, describe data sources identified in the review, set inclusion and exclusion criteria for the study, describe data extraction process, assess quality measures for the systematic review, and describe statistical procedures for the quantitative analysis. We aim to address our key research question that whether pharmacist intervention is effective on improving HRQoL and clinical outcomes (in terms of reduced hospitalization and mortality) among patients with HF compared to usual/standard care.

\section{Method}

\section{Study protocol}

In order to include all relevant information the Preferred Reporting Items for Systematic Reviews and Meta-Analyses Protocols (PRISMA-P) checklist will be employed as a tool [27]. The PRISMA flow diagram will be used for identification, screening, eligibility and inclusion of studies at different stages of the review process (www.prisma-statement.org). The review is approved in the International Prospective Register of Systematic Reviews (PROSPERO) with registration ID CRD42020158236. 


\section{Research question}

$\checkmark$ Is pharmacist intervention effective on health related quality of life among patients with HF compared to usual/standard care?

$\checkmark$ Is pharmacist intervention improves clinical outcomes (reduces patient hospitalization and mortality) among patients with HF compared to usual/standard care?

We believe these questions are highly relevant to policy makers and service providers. Our review will focus on the journals that are randomized controlled trial only.

\section{Search strategy and data source}

Databases indexed in Medline (PubMed), Embase, Cochrane Central Register of Controlled Trials (CENTRAL), Scopus and Google scholar will be conducted to retrieve journals. Original peer reviewed articles published in English language from 1990 to December, 2019 will be identified using the terms heart failure, pharmacist intervention, health related quality of life and clinical outcomes. In our search medical subject heading (MeSH) and Boolean operators will be employed to search journals using 'heart failure' (MeSH) OR 'cardiac failure' (MeSH) AND 'pharmacist intervention' OR 'clinical pharmacist intervention' OR 'pharmaceutical care' AND 'health related quality of life' OR 'quality of life' AND 'clinical outcome' OR 'health outcome' OR 'patient outcome'. We limited the studies to randomized controlled trials using the term 'randomized controlled trial'. Titles of all retrieved studies will be screened and abstracts of the selected articles will be further evaluated. Article screening and the selection process will be carried out by the primary author and then independently reviewed by two other authors.

\section{Eligibility criteria}

Studies that reported pharmacist intervention and provided data on health related quality of life (or quality of life) and clinical outcomes (mortality, hospitalization) will be included in this review. Additionally, studies that reported pharmacist-led multidisciplinary team interventions will be included. The following studies will be excluded: case reports and case series, case-controls, cohorts, observational and non-research articles and commentaries. Abstracts from conference and journals whose full-text cannot be retrieved will be excluded. In addition, studies that will not report primarily pharmacist intervention such as nurse-led multi-disciplinary team interventions, interventions requiring telephone monitoring, telemedicine, and web-based interventions will be excluded. Therefore, original peer-reviewed articles of randomized controlled trial that report pharmacist intervention and consider HRQoL and clinical outcomes among patients with HF will be eligible for inclusion.

\section{Data extraction}


Data will be extracted by one author and will be approved by other two authors independently. Any discrepancy will be resolved by agreement. The following information will be extracted from included studies: name of first author, year of publication, study setting/country, study design, number of participants, follow-up/duration, type of intervention and main outcomes of the study. For data management, we will use the ENDNOTE reference software version 7 (Thomson Reuters, Stamford, CT, USA) in title review, abstract screening, and removal of duplicates.

\section{Quality assessment}

Studies will be reviewed independently to assess the quality of included studies to minimize the risk of bias using the modified Downs and Black checklist designed to evaluate both randomized controlled trials and nonrandomized studies. The check list contains 27 items covering quality of reporting, confounding, bias, and representativeness of sample size [28].

\section{Data synthesis and analysis}

We will analyze data in accordance with the Cochrane handbook [29]. We will pool data from studies which are sufficiently similar. Meta-analysis will be performed when we find at least two studies that report the same HRQoL and clinical outcome measures to see the pooled effect of pharmacist intervention. When this is not possible due to insufficient number of studies, we will conduct narrations of the study results. Heterogeneity of included studies will be assessed using the standard Cochrane $Q$ statistics and the $R$ statistics. Publication bias will be assessed using funnel plots, and forest plot will be constructed using a random effect model. Because of variability in reporting among studies, standardized mean differences will be used as an estimate of the effect size. Analysis for the dichotomous outcome studies will be converted into standardized mean difference and will be presented with $95 \%$ confidence intervals. The analyses will be conducted using the Cochrane Review Manager (RevMan) V.5.3 software (Copenhagen: The Nordic Cochrane Centre, The Cochrane Collaboration, 2014). The study will be reported utilizing Preferred Reporting Items for Systematic Reviews and Meta-Analyses Protocols (PRISMA-P) 2015 statement [27].

\section{Discussion}

Heart failure is a syndrome associated with higher rates of hospitalization, morbidity, mortality and poor quality of life. Pharmacist interventions have improved quality of life on specific diseases; however, the systematic review and meta-analysis study of pharmaceutical care intervention on HRQoL proved no statistically significant impact of pharmaceutical care intervention on HF [19]. Currently there are important gaps on the effectiveness of pharmacist intervention. We believe this review will provide comprehensive evidence on the effectiveness of pharmacist intervention among patients with HF. Currently we have screened more than 1820 abstracts and identified more than 145 potential studies which we will intend to retrieve their full text review. 
We are updating the review process and the PRISMA file is under development. Our search yielded more than 660 articles of these 306 were excluded based on titles and abstracts. Currently we identified more than 30 potential journals to be included for analysis.

\section{Acronyms}

HF: Heart Failure; HRQoL: Health Related Quality of Life; NYHA: New York Health Association; PRISMA-P: Preferred Reporting Items for Systematic Reviews and Meta-Analyses Protocols; QoL: Quality of Life.

\section{Declarations}

\section{Acknowledgement}

We would like to acknowledge Mr. Fekede (Asst. prof...) for the contribution made in providing journals from indexing database (EMBASE).

\section{Ethics approval and consent to participate}

Not applicable

\section{Consent for publication}

Not applicable

\section{Availability of data and material}

Not applicable

\section{Competing interests}

The authors declare that they have no competing interests.

\section{Funding}

None

\section{Authors' contributions}


MTT and ABB designed the study, led the search strategy for abstraction, plans for data extraction, and drafted the first version of the manuscripts with significant inputs from EE, HBA and TTG. All authors have given final approval of the version to be published.

\section{References}

1. Yancy CW, Jessup M, Bozkurt B, Butler J, Casey DE, Colvin MM, Drazner MH, Filippatos GS, Fonarow GC, Givertz MM: 2017 ACC/AHA/HFSA focused update of the 2013 ACCF/AHA guideline for the management of heart failure: a report of the American College of Cardiology/American Heart Association Task Force on Clinical Practice Guidelines and the Heart Failure Society of America. Circulation 2017, 136(6):e137-e161.

2. Ponikowski P, Voors A, Anker S, Bueno H, Cleland J, Coats A, Falk V, González-Juanatey J, Harjola V, Jankowska E: Authors/Task Force Members; Document Reviewers. 2016 ESC Guidelines for the diagnosis and treatment of acute and chronic heart failure: The Task Force for the diagnosis and treatment of acute and chronic heart failure of the European Society of Cardiology (ESC). Developed with the special contribution of the Heart Failure Association (HFA) of the ESC. European journal of heart failure 2016, 18(8):891-975.

3. Spoletini I, Vitale C: Clinical diagnosis in heart failure. In: International Cardiovascular Forum Journal: $2017 ; 2017$.

4. Zannad F, Garcia AA, Anker SD, Armstrong PW, Calvo G, Cleland JG, Cohn JN, Dickstein K, Domanski MJ, Ekman I: Clinical outcome endpoints in heart failure trials: a European Society of Cardiology Heart Failure Association consensus document. European journal of heart failure 2013, 15(10):10821094.

5. Ponikowski P, Anker SD, AlHabib KF, Cowie MR, Force TL, Hu S, Jaarsma T, Krum H, Rastogi V, Rohde LE: Heart failure: preventing disease and death worldwide. ESC Heart Failure 2014, 1(1):4-25.

6. McKelvie R: Heart failure, search date August 2010. BMJ Clinical Evidence Available online: http://www clinicalevidence com 2011.

7. Chaudhry SI, McAvay G, Chen S, Whitson H, Newman AB, Krumholz HM, Gill TM: Risk factors for hospital admission among older persons with newly diagnosed heart failure: findings from the Cardiovascular Health Study. Journal of the American College of Cardiology 2013, 61(6):635-642.

8. Kinugasa Y, Kato M, Sugihara S, Yanagihara K, Yamada K, Hirai M, Yamamoto K: Multidisciplinary intensive education in the hospital improves outcomes for hospitalized heart failure patients in a Japanese rural setting. BMC health services research 2014, 14(1):1.

9. Sui X, Gheorghiade M, Zannad F, Young JB, Ahmed A: A propensity matched study of the association of education and outcomes in chronic heart failure. International journal of cardiology 2008, 129(1):93-99.

10. McNaughton CD, Cawthon C, Kripalani S, Liu D, Storrow AB, Roumie CL: Health literacy and mortality: a cohort study of patients hospitalized for acute heart failure. Journal of the American Heart Association 2015, 4(5):e001799. 
11. Macabasco-O'Connell A, DeWalt DA, Broucksou KA, Hawk V, Baker DW, Schillinger D, Ruo B, BibbinsDomingo K, Holmes GM, Erman B: Relationship between literacy, knowledge, self-care behaviors, and heart failure-related quality of life among patients with heart failure. Journal of general internal medicine 2011, 26(9):979-986.

12. Heo S, Moser DK, Lennie TA, Zambroski CH, Chung ML: A comparison of health-related quality of life between older adults with heart failure and healthy older adults. Heart \& Lung: The Journal of Acute and Critical Care 2007, 36(1):16-24.

13. Juenger J, Schellberg D, Kraemer S, Haunstetter A, Zugck C, Herzog W, Haass M: Health related quality of life in patients with congestive heart failure: comparison with other chronic diseases and relation to functional variables. Heart 2002, 87(3):235-241.

14. Milosevic DP, Markovic I, Brajovic MD, Bojovic O, Potic B, Davidovic M: Health-related quality of life in elderly patients hospitalized with chronic heart failure. Clinical interventions in aging 2013, 8:15391546.

15. Allen LA, Gheorghiade M, Reid KJ, Dunlay SM, Chan PS, Hauptman PJ, Zannad F, Konstam MA, Spertus JA: Identifying patients hospitalized with heart failure at risk for unfavorable future quality of life. Circulation: Cardiovascular Quality and Outcomes 2011, 4(4):389-398.

16. Bisharat B, Hafi L, Baron-Epel O, Armaly Z, Bowirrat A: Pharmacist counseling to cardiac patients in Israel prior to discharge from hospital contribute to increasing patient's medication adherence closing gaps and improving outcomes. J Trans/ Med 2012, 10(1):34.

17. Hatah E, Braund R, Tordoff J, Duffull SB: A systematic review and meta-analysis of pharmacist-led fee-for-services medication review. British journal of clinical pharmacology 2014, 77(1):102-115.

18. Mekonnen AB, McLachlan AJ, Jo-anne EB: Effectiveness of pharmacist-led medication reconciliation programmes on clinical outcomes at hospital transitions: a systematic review and meta-analysis. BMJ open 2016, 6(2):e010003.

19. Mohammed MA, Moles RJ, Chen TF: Impact of pharmaceutical care interventions on health-related quality-of-life outcomes: a systematic review and meta-analysis. Annals of Pharmacotherapy 2016, 50(10):862-881.

20. Herring H, Smith W, Ripley T, Farmer K: Pharmacist Involvement at Discharge with The Joint Commission Heart Failure Core Measure: Challenges and Lessons Learned. Hospital pharmacy 2014, 49(11):1017.

21. Lee VW, Choi LM, Wong WJ, Chung HW, Ng CK, Cheng FW: Pharmacist intervention in the prevention of heart failure for high-risk elderly patients in the community. BMC cardiovascular disorders 2015, 15(1):1.

22. Chua SS, Kok LC, Yusof FAM, Tang GH, Lee SWH, Efendie B, Paraidathathu T: Pharmaceutical care issues identified by pharmacists in patients with diabetes, hypertension or hyperlipidaemia in primary care settings. BMC health services research 2012, 12(1):1.

23. Eggink RN, Lenderink AW, Widdershoven JW, van den Bemt PM: The effect of a clinical pharmacist discharge service on medication discrepancies in patients with heart failure. Pharmacy world \& 
science 2010, 32(6):759-766.

24. Truong JT, Backes AC: The impact of a Continuum of Care Resident Pharmacist on heart failure readmissions and discharge instructions at a community hospital. SAGE open medicine 2015, 3:2050312115577986.

25. Sadik A, Yousif M, McElnay J: Pharmaceutical care of patients with heart failure. British journal of clinical pharmacology 2005, 60(2):183-193.

26. Holland R, Battersby J, Harvey I, Lenaghan E, Smith J, Hay L: Systematic review of multidisciplinary interventions in heart failure. Heart (British Cardiac Society) 2005, 91(7):899-906.

27. Moher D, Shamseer L, Clarke M, Ghersi D, Liberati A, Petticrew M, Shekelle P, Stewart LA: Preferred reporting items for systematic review and meta-analysis protocols (PRISMA-P) 2015 statement. Systematic reviews 2015, 4(1):1.

28. Downs SH, Black N: The feasibility of creating a checklist for the assessment of the methodological quality both of randomised and non-randomised studies of health care interventions. Journal of Epidemiology \& Community Health 1998, 52(6):377-384.

29. Higgins JP, Altman DG, Gøtzsche PC, Jüni P, Moher D, Oxman AD, Savović J, Schulz KF, Weeks L, Sterne JA: The Cochrane Collaboration's tool for assessing risk of bias in randomised trials. $B M J$ (Clinical research ed) 2011, 343:d5928. 